\title{
Transrectal Ultrasound
}

National Cancer Institute

\section{Source}

National Cancer Institute. Transrectal Ultrasound. NCI Thesaurus. Code C18315.

A procedure that uses sound waves to create a video image of the pelvic organs and tissues. A small, lubricated probe placed into the rectum releases sound waves, which create echoes as they enter a pelvic organ. The echoes that bounce back are sent to a computer that translates the pattern of echoes into a picture of the specific organ under examination. This procedure is most often used to evaluate the prostate gland. 